\title{
KanAK ATtAK! HipHop Und (ANTi-)IdentitätSMOdELle DER „ZWEITEN GENERATION“ 1
}

\author{
Fatima El-Tayeb ${ }^{2}$
}

„Das ist wie zwei Sprachen zu sprechen, damit müssen wir halt irgendwie [leben]. Das ist das, was wir akzeptieren müssen: Widersprüche. Es gibt mehr Widersprüche am Tag, als man glaubt. Ich repräsentiere Widerspruch und in HipHop ist auch viel Widerspruch drin... Ich finde aber, das macht diese Sache so interessant, gerade weil sie so irgendwo dazwischen rumhängt."“

Torch, Advanced Chemistry (Menrath 2001: 117)

\section{Vom Gastarbeiter-Diskurs zum Hybrid-Hype zum Gastarbeiter-Diskurs...}

„Border communities“, „Hybride Kulturen“ und „multikulturelle Subjekte“ sind in, in den populären Medien ebenso wie im akademischen Diskurs. ${ }^{3}$ In Deutschland verlagerte sich die Diskussion in den letzten zehn Jahren von einem problemorientierten Blick auf „Gastarbeiter“ und „benachteiligte Jugendliche“ - eine Diskussion, die von sozialwissenschaftlichen Ansätzen geprägt war - hin zu einer zelebrierenden Präsentation hipper, hybrider, erfolg-

1 „Zweite Generation“ steht hier, wie im Laufe des Texts deutlich werden wird, für die relativ heterogene Gruppe derjenigen, die zwischen die strikten diskursiven Modelle von „,deutsch“ und „fremd“ fallen, deutsche ,sichtbare Minderheiten,“ Binationale, MigrantInnen, in Deutschland Geborene ohne deutschen Pass usw. Eine Gruppe, die als solche noch kaum wahrgenommen und benannt wird, weswegen mir der zwar nicht wirklich zutreffende, immerhin aber gebräuchliche Begriff „Zweite Generation“ an dieser Stelle vertretbar und sinnvoll scheint.

2 Eine frühere Fassung dieses Textes erschien in: Eder, Angelika (2003): ,Wir sind auch da!' Über das Leben von und mit Migranten in europäischen Großstädten, Hamburg, Dölling und Galitz, 2003.

3 Siehe zur Definition von Hybridität und Multikulturalismus insbesondere die gesammelten Schriften Stuart Halls (seit 1989) und Bhabha 2000. Zu einer Darstellung der im anglophonen Raum kontrovers geführten Debatte um diese Begriffe: Bronfen 1997 und Breger, in: Röttger/Paul 2000. 
reicher junger Autoren, Regisseure oder Rapperinnen. ${ }^{4}$ Es ist zweifellos ein Fortschritt, dass „Fremde“ in Deutschland inzwischen in einigen Kontexten als aktiv Handelnde statt als bloße Objekte soziologischen Interesses wahrgenommen werden, dass das „Hybride“, „Zwischen den Kulturen Stehende“ als Vorteil und Bereicherung in einer zunehmend globalisierten Welt präsentiert wird. So befindet etwa auch die taz unter der Überschrift „Bastarde gewinnen!“ durchaus zustimmend: „Der Hybride, der Mischling, der Entwurzelte, der Grenzgänger, der Bastard hat Hochkonjunktur. Er verkörpert Offenheit, Flexibilität, Anpassungsfähigkeit, Kreativität, eben all das, was eine globalisierte Wirtschaft braucht" (Kresta 2001: 15). Während die allgemeine Begeisterung für „den Hybriden“ so als Fakt präsentiert wird, herrscht jedoch gleichzeitig merkwürdig wenig Interesse an ihm als realem Subjekt, an den sozialen und ökonomischen Konsequenzen der Globalisierung (einschließlich der europäischen Vereinigung) für Gemeinschaften von MigrantInnen und ethnische Minderheiten in Deutschland. Das jüngste Medieninteresse an der „Zweiten Generation“ scheint kaum mehr als eine erneute Objektifizierung, eher hippes Saison-Thema als echtes Interesse an den Lebensumständen dieser neuentdeckten Gruppe.

Dieses Desinteresse überrascht umso mehr angesichts einer seit über zehn Jahren stetig zunehmenden rassistischen Gewalt in Deutschland und Europa. Der deutschen Debatte um diese Gewalt gelang und gelingt es, eine Beteiligung der (potenziellen) Opfer fast gänzlich zu vermeiden. ${ }^{5}$ In diesem weniger hippen Kontext, der nichtsdestoweniger von zentraler Bedeutung für dieses Land ist, verschwindet das hybride Subjekt sang- und klanglos, und alle, die nicht in das dominante Raster des „Deutschen“ passen, mutieren wieder zum stummen, „fremden“ Objekt. Diese Fremdheit, so scheinbar die stillschweigende Übereinkunft, disqualifiziert es für einen Platz im öffentlichen Raum diskursiv und auch sonst. ${ }^{6}$ Dass dieser Ausschluss aus der deutschen Debatte weitestgehend unhinterfragt (zumindest von mehrheitsdeutscher Seite aus) vonstatten gehen kann, hat viel damit zu tun, dass das ,multikulturelle Sub-

4 Siehe u.a. die Beiträge zu den Titelthemen „Gefährlich fremd. Das Scheitern der multikulturellen Gesellschaft“, in: Der Spiegel 16 (1997); „Fremd und Deutsch. Warum die Integration von Ausländern Unsinn ist", in: Spiegel Reporter 2 (2000). Außerdem: Kresta 2001: 15.; Bax 2001: X-XI.

5 Wenn überhaupt daran gedacht wird, das Gespräch mit Betroffenen zu suchen, ist es fast immer Paul Spiegel, Vorsitzender des Zentralrats der Juden in Deutschland, der in der öffentlichen Debatte als einsamer Vertreter des ,Anderen" gecastet wird. Sein Versuch, dem entgegenzuwirken, indem er immer wieder auf die (ethnische) Heterogenität der deutschen Gesellschaft verweist, bleibt angesichts seiner isolierten Position im Mehrheitsdiskurs notgedrungen ohne große Wirkung.

6 Siehe etwa den neugegründeten Hamburger „Integrationsbeirat,“ der die Aufgaben der am 30.6.2002 „,verabschiedeten“ Ausländerbeauftragten übernommen hat. Ihm gehören zwar zahlreiche Prominente ohne jegliche Sachkenntnis an, VertreterInnen einiger der größten Hamburger Migrantengruppen werden jedoch nicht beteiligt. Dierbach 2002: 25. 
jekt" in Deutschland als geschichtslos erscheint; ins Leben gerufen durch die liberale deutsche Gesellschaft, nicht durch sein eigenes Insistieren und Resistieren. Es ist nicht so, dass dieser Subalterne nicht sprechen könnte, eher ist es die dominante Gesellschaft, die vorgibt, die barbarischen Laute nicht zu verstehen.

\section{Störende Stimmen I: Kanak Attak}

Eine Organisation, die einiges Aufsehen verursachte, indem sie die zum Schweigen gebrachte Stimme mitten im mainstream ertönen ließ, ist Kanak Attak, ein loser Zusammenschluss von MigrantInnen, zweiter Generation und anderen. Diese zugegebenermaßen vage Gruppenbeschreibung ergibt sich aus Kanak Attaks Strategie, in der das Nicht-Festlegen auf klare Begrenzungen und ethnische Kategorisierung bei gleichzeitiger Referenz auf gemeinsame Erfahrungen der Ausgrenzung, mit all seinen Schwierigkeiten, Programm ist:

„[W]eil Kanak Attak eine Frage der Haltung und nicht der Herkunft oder der Papiere ist, sind auch Nichtmigranten und Deutsche der $\mathrm{n}^{2}$-Generation mit bei der Sache. Aber die bestehende Hierarchie von gesellschaftlichen Existenzweisen und Subjektpositionen lässt sich nicht spielerisch überspringen. Es sind eben nicht alle Konstruktionen gleich. Damit bewegt sich das Projekt zwischen auflösbaren Widersprüchen, was das Verhältnis von Repräsentation, Differenz und die Zuschreibung ethnischer Identitäten angeht“" (Kanak Attak Manifest 1998).

Die Gruppe wendet sie sich gegen kulturellen hybriden Chic und ausschließenden sozialen Essentialismus als zwei Seiten derselben Medaille. Kanak Attak kritisiert eine Identitätspolitik, die nicht nur „Deutsche“ und „Migranten" voneinander abgrenzt, sondern auch letztere anhand dubioser Definitionen von Legitimität und Legalität spaltet, und präsentiert stattdessen eine „Kanaken“-Identität, die einerseits Minderheiten über ethnisierte Grenzen miteinander verbindet, andererseits wohlmeinende mehrheitsdeutsche Konstrukte vom ,ausländischen Mitbürger“ bis zur unterdrückten Migrantin zurückweist. Der Versuch, „,Zwischen auflösbaren Widersprüchen“ einen strategisch flexiblen, politisch wirksamen Anti-Essentialismus zu praktizieren, gehört zu den interessantesten Strategien Kanak Attaks (auch wenn sich inzwischen teilweise deutlicher Frust mit diesem Modell breit macht).$^{7}$ Wo genau

7 „Am Anfang war das einer unserer meistgeäußerten Sätze: Identitäten durchkreuzen zu wollen. Das ist mir mittlerweile so fern, ein abstraktes Gerede, das nirgendwohin führt. Eine Selbsttäuschung, in der politische Fragen so weit subjektiviert werden, dass man glaubt, wenn man im Alltag verschiedene Identitäten zurückweist oder verwischt, habe man schon eine widerständige Praxis. Das ist ein Baustein, der zwar interessant sein kann, für sich aber nicht ausreicht, weil das Umbauen von Identitäten ständig und auf allen Ebenen geschieht und als solches nicht notwendigerweise widerständig ist. Identitäten durchkreuzen, das tun alle“ (Bojadzijev 2001: 14). 
die Gruppe sich im steinigen Feld postkolonialer anti-essentialistischer Politik positioniert, ist so nicht immer deutlich (und auch nicht konsistent), unumstritten war jedoch stets die zentrale Rolle, die Geschichte im Politikverständnis von Kanak Attak einnimmt:

„Eines unserer Ziele war, die Geschichte des antirassistischen Widerstands von MigrantInnen zu rekonstruieren, eine ungeschriebene und auch unbekannte Geschichte. Wir wollten eine Tradition freilegen, die zum Teil nur untergründig gewirkt hat und verschüttet war. Die Idee war: Wenn du eine eigene Geschichte hast, bist du machtvoller, kannst du auf etwas verweisen, was überliefert und angeeignet werden kann“ (Bojadzijev 2001: 14).

Entsprechend war das bisher größte Kanak Attak-Projekt eine Revue, die um 50 Jahre migrantischen Widerstand in der Bundesrepublik kreiste. ${ }^{8}$ In der breiten Öffentlichkeit jedoch wird, trotz aller Berichte zum 40jährigen Jubiläum des Anwerbeabkommens mit der Türkei, diese Migrationstradition immer noch nicht als Teil der deutschen Geschichte begriffen.

\section{Deutschland ist (k)ein Einwanderungsland}

Nur sehr langsam hat sich ein gesellschaftliches Eingeständnis der Tatsache entwickelt, dass Deutschland ein „Einwanderungsland“ ist, und nach wie vor gibt es kaum ein Bewusstsein der Tradition von Migration, die Jahrhunderte vor den ersten „Gastarbeiter“-Abkommen in den 1950ern begann. ${ }^{9}$ In der vergangenen Dekade war das Dogma von Deutschland als einem NichtEinwanderungsland ein solch dominanter Bestandteil von Diskussionen um Staatsbürgerschaft, Migration und rechtsradikale Gewalt, dass es nahe liegt anzunehmen, es sei von zentraler Bedeutung für das deutsche nationale Selbstverständnis. Offensichtlich steht hier viel mehr auf dem Spiel als juristi-

8 Im April 2001 an der Berliner Volksbühne. Ein ähnliches Projekt, das sich nicht mehr nur mit Arbeitsmigration, sondern vor allem auch die Legalisierung von Menschen ohne Aufenthaltsstatus zum Thema hatte, fand im Mai/Juni 2002 in Berlin und Frankfurt/Main statt.

9 Ebenso sehr scheint in Vergessenheit geraten zu sein, dass im 18. und 19. Jahrhundert kaum ein Volk so viele „Zuwanderer“ produzierte wie die Deutschen. Zumindest innerhalb des wissenschaftlichen Diskurses verliert das Thema Migration jedoch langsam seinen traditionellen Außenseiterstatus. Trotz einiger wichtiger Veröffentlichungen - z.B. zahlreiche Titel von Klaus J. Bade und seinem Osnabrücker Institut für Migrationsforschung und Interkulturelle Studien; auch: Herbert 2001; Motte/Ohliger/von Oswald 1999 - ist die Migrationsforschung in Deutschland noch entwicklungsbedürftig, vor allem da MigrantInnen - analog zur gesamtgesellschaftlichen Haltung - nach wie vor meist lediglich als passive Untersuchungsobjekte betrachtet werden. Wichtige Ausnahmen sind u.a. Ha 1999; Gelbin/Konuk/Piesche 1999. 
sche Konstruktionen. ${ }^{10}$ Dennoch verabschiedet Deutschland sich unbestreitbar, wenn auch zögernd, von einer mono-ethnischen Nicht-EinwanderungsIdentität. Die überfällige Einführung von ius soli-Elementen in das Staatsbürgerschaftsrecht und der Versuch, Migration gesetzlich zu regeln (damit zugebend, dass sie existiert), zeigen, dass eine Trennung von Nationalität und Ethnizität im deutschen Identitätskonzept möglich ist. Die erbitterten Debatten um diese begrenzten Zugeständnisse an eine eher kulturelle denn biologistische Definition nationaler Identität zeigen aber auch, dass dieser Prozess weder automatisch voranschreiten wird noch unumkehrbar ist (schließlich erklärte der bayerische Ministerpräsident und spätere Kanzlerkandidat der CDU/CSU Edmund Stoiber schon zwei Jahre vor dem 11. September 2001, die doppelte Staatsbürgerschaft gefährde die innere Sicherheit mehr als der Terrorismus der RAF in den 1970er Jahren). ${ }^{11}$ In der Wahrnehmung wie in der Selbstdarstellung derjenigen, die sichtbare Produkte der verleugneten Migrationsgeschichte sind, existieren der dominante Diskurs von Deutschland als einem traditionellen „Nicht-Einwanderungsland“ und die - oft verschütteten Gegen-Diskurse einer tatsächlichen Diversität spannungsreich nebeneinander. Eine Verschmelzung bzw. Transformation der Kategorien „,deutsch“ und „fremd“ wird trotz kulturalistischem Hybrid-Hype, der ja oberflächlich genau diese „Verschmelzung“ feiert, kaum gedacht. Stattdessen wird eine „InnenAußen"-Perspektive konstruiert, die Gemeinsamkeiten und Überschneidungen nicht zulässt.

Traditionell beinhaltet dieses Modell auch die Etablierung geografischer Gegensätze: dort das migrantische Arbeiterviertel, das ausschließlich als Problemgebiet wahrgenommen und der hier herrschenden mehrheitsdeutschen, bürgerlichen Normalität eines stabilen, aber von zunehmender Angst um die

10 Was genau auf dem Spiel steht, ist natürlich eine äußerst interessante und wichtige Frage, deren Beantwortung an dieser Stelle jedoch nicht versucht wird, da es mir hier um Eigendefinitionen marginalisierter Gruppen geht. Ich verweise u.a. auf El-Tayeb 2001: 72-81.

11 Unterschriftenaktion zur Ausländerpolitik. Union macht gegen Bonn mobil. Stoiber: Doppelte Staatsbürgerschaft gefährlicher als RAF, in: Süddeutsche Zeitung, 04. 01. 1999, S. 5. Während Stoibers kaum noch implizite Gleichsetzung von Migration und Terrorismus 1999 noch etwas aus dem Rahmen fiel, ist sie im Zuge der staatlichen Reaktionen auf die Anschläge vom 11. September nahezu zum Gemeinplatz geworden. Die Attentate wurden weltweit zum Anlass genommen, Bürgerrechte im Namen der ,inneren Sicherheit“ zu beschneiden und gleichzeitig eine repressive Ausländergesetzgebung als probates Mittel im Kampf gegen den internationalen Terrorismus zu verkaufen. Insbesondere moslemische Migranten stehen pauschal unter Tatverdacht. Der neue Konsens, auf den sich das Feindbild Islam stützen kann, zeigt sich im europaweiten Siegeszug rechtsextremer Parteien, deren Programm sich oft auf explizite Ausländer- und Islamfeindlichkeit beschränkt, aber etwa auch darin, dass Oriana Fallacis rassistische Hasstiraden, die einen heiligen Krieg gegen alle „Söhne Allahs“" halluzinieren (Fallaci 2002), sowohl in der linksradikalen „Bermuda“ als auch der rechtskonservativen „Welt“ abgedruckt werden. 
„innere Sicherheit“ bedrohten Mittelstandsviertels gegenübergestellt wird. Dies ist eine Konstruktion, die der Realität nicht unbedingt gänzlich widerspricht, sie aber auch sicher nicht neutral widerspiegelt. ${ }^{12}$ In den postindustriellen urbanen Zentren der kriselnden new economy wird die Künstlichkeit dieser Polarisierung zunehmend deutlich, Widersprüche und Komplexitäten treten zutage. Im Hamburger Schanzenviertel etwa, um ein Beispiel von vielen herauszugreifen, koexistieren Multikulti-Schick, der Yuppies und Touristen anzieht, erfolgreiche migrantische Kleinunternehmer, Werbefirmen, radikaler linker Widerstand, ethnische Selbstorganisation, offene Drogenszene und eine rassistische Behördenpolitik gegenüber pauschal als Drogendealern eingestuften so genannten „Schwarzafrikanern“, die auch von breiten Teilen der Einwohnerschaft getragen wird. Wird das Schanzenviertel über die Stadtteilgrenzen hinaus wahrgenommen, ist es allerdings zumeist allein dieser letzte Punkt, der das Zusammenleben zu charakterisieren scheint: Nicht-mehrheitsdeutsche Bevölkerungsgruppen werden als Außenseiter, Eindringlinge präsentiert, die die ausgewogene deutsche Normalität aus dem Gleichgewicht bringen. Seien es „schwarzafrikanische Dealer“, die für das Drogenproblem verantwortlich gemacht werden, oder - wie vor zehn Jahren - Roma, die für den Einzug von Gesetzlosigkeit, Kriminalität und Gewalt standen: der angeblich kausale Zusammenhang von „Problemgebiet“ und ethnischer Andersartigkeit bleibt bestehen. ${ }^{13}$ Zwar gibt es expliziten Widerstand aus der Alternativkultur gegen diese Ausgrenzung, die Außensicht bleibt dennoch bestehen. Auch in der „anti-rassistischen“ linken Szene mangelt es an „Dialogkultur“; die tatsächliche Verschiebung der Grenzen, die reale Pluralität wird nicht gespiegelt.

\section{Störende Stimmen II: HipHop und nationale Identität}

Menschen, die zwar ihr ganzes oder den überwiegenden Teil ihres Lebens in Deutschland verbracht haben, aber dennoch nicht weiß, deutsch und christlich sind (wenn auch vielleicht manches davon), stellen in urbanen Gebieten wie

12 Eine Renaissance erlebte dieses Modell nach der Veröffentlichung der PISAStudie, bei deren Vergleich der Leistungsfähigkeit von Schulkindern Deutschland ausgesprochen schlecht abschnitt. Schnell wurde die Erklärung im (zu) hohen „Ausländeranteil“ an innerstädtischen Schulen gesucht. Siehe Althaus et al. 2002.

13 Ariane Barths Artikel „Hier steigt eine Giftsuppe auf,“ in: Der Spiegel 42 (1991), über den „Terror“ von Roma-Kindern im Hamburger Karolinenviertel, führte zu einer langanhaltenden Auseinandersetzung innerhalb der Presse, zunächst lokal, dann national, mit der „Bedrohung“ durch Roma einerseits und der medialen Konstruktion von Stereotypen andererseits. Vgl. Schröder 2000. Im Hamburger Wahljahr 2001 berichteten die großen lokalen Tageszeitungen fast täglich von steigender „Ausländerkriminalität“ und ,schwarzafrikanischen Dealern“, damit die „Hamburg - Hauptstadt des Verbrechens“-Kampagne des späteren Wahlsiegers Ronald Schill speisend und gleich die Schuldigen identifizierend. 
dem Hamburger Schanzenviertel jedoch einen substanziellen Teil der Bevölkerung. Entsprechend konnte sich dort im letzten Jahrzehnt ein Gegendiskurs entwickeln, der um eine Diaspora-Identität kreist, die zwar den Rückbezug auf die Herkunftsländer (der Vorfahren) und andere diaspora communities beinhaltet, sich aber nicht mit ihnen gleichsetzt. Stattdessen wird ein anderes Modell nationaler Identität entwickelt, das die rund eine Million in Deutschland geborenen „TürkInnen“ einschließt, die ca. 500.000 schwarzen Deutschen, die zumeist als „AmerikanerInnen“ oder „AfrikanerInnen“ wahrgenommen werden, oder die 70.000 deutschen Sinti, um nur einige derjenigen zu nennen, die nicht mitgedacht werden, wenn ,deutsch“ gesagt wird. „Gegendiskurs“ heißt hier für mich, dass es nicht um den Versuch von Einzelnen oder Organisationen geht, in den dominanten Diskurs zu intervenieren und sich seiner Mechanismen anzupassen, sondern um eine eigenständige Auseinandersetzung marginalisierter Gruppen, die auf der Situation in der Bundesrepublik basiert, aber andere Bezugspunkte und Vorbilder hat.

Unter diesen wiederum nimmt die HipHop-Kultur eine zentrale Position ein. Die erste Generation von jugendlichen MigrantInnen organisierte sich meist entlang ethnischer Grenzen und in traditionellen politischen Strukturen; Gewerkschaften spielten aufgrund der Beschäftigungsstruktur eine wichtige Rolle, ebenso (feministische) Stadtteilarbeit. Die Situation der „Zweiten Generation“ als „fremd im eigenen Land“, mit einer Orientierung nicht auf „Herkunfts-“ und potenzielles „Rückkehrland“, sondern auf die bundesrepublikanische Gegenwart, in der sie meist als Störfaktor wahrgenommen wird, schafft eine Affinität zu HipHop - aufgrund der dominanten Rolle, die Rap innerhalb der internationalen Jugendkultur spielt, aber auch, weil er ein direktes Produkt der beschriebenen urbanen Situation ist. Gruppen wie die afro-italo-deutschen Advanced Chemistry, Pioniere nicht nur des deutschsprachigen HipHop, sondern mit „Fremd im eigenen Land“ von 1992 auch des Diskurses, um den es mir hier geht, benutzten das synkreti[sti]sche Potenzial des HipHop, um eine geleugnete Identität zu benennen. ${ }^{14}$ Aufbauend auf der afro-amerikanischen Widerstandstradition, ist HipHop ein Produkt der Interaktion verschiedener communities of color, kreative Reaktion auf die zunehmende Subproletarisierung der, in der Hauptsache nicht-weißen, innerstädtischen Bevölkerung in den USA. ${ }^{15}$ Nun zeigt sich sicher gerade in der Geschichte der Popularisierung afro-amerikanischer Musik, dass die Aneignung eines Musikstils keine

14 Synkretismus fokussiert im Gegensatz zum Hybriditätsmodell, in dessen Gespaltenheitstopos auch immer Anklänge an die „tragic mulatto“-Tradition mitschwingen, auf das kreative Potenzial von Verschmelzungsprozessen. Siehe u.a. Hall/Morley/Kuan-Hsing 1996 und Bronfen 1997.

15 In den USA führten die Massenabwanderung der Industrie, umfassende Kürzungen im Sozialbereich und wachsende Einnahmeverluste der Kommunen durch die Abwanderung des Mittelstands in die Vororte in den 1980ern zu einer Verelendung der innerstädtischen Bevölkerung, die wiederum von der Gesamtgesellschaft mehr oder weniger abgeschrieben und sich selbst überlassen wurde. Vgl. Kelly 1997. 
Identifizierung mit seinen ursprünglichen Inhalten verlangt. Die Möglichkeiten, die HipHop für Deutschland bot, liegen jedoch auf der Hand: der Ausschluss der Nicht-Mehrheitsbevölkerung aus dem gesellschaftlichen Diskurs ist hier besonders extrem. HipHop bot eine Plattform für Marginalisierte und schuf so ein Bewusstsein der gemeinsamen Lage. Advanced Chemistry beziehen sich im Covertext ihrer zweiten Single, „Welcher Pfad führt zur Geschichte" (1993), explizit auf diese urbane Diaspora-Tradition und versuchen gleichzeitig, bisher noch nicht als Gemeinschaft gedachte Gruppen in Deutschland in diese Tradition einzuschließen:

„Jeder Aktivist und jede Aktivistin der heutigen HipHop-Szene, ob in Bremerhaven oder Brooklyn, steht in der Tradition der Zulu Nation, ganz gleich, ob er oder sie es anerkennt oder nicht (...). In New York waren die Innovatoren dieser Kultur überwiegend Afroamerikaner, Jamaikaner, Haitianerinnen, Puertorikaner ... Es ist kein Zufall, daß sich auch in Deutschland wegen des rebellischen Inhalts dieser Kultur gerade viele Schwarze Deutsche, Türken und Kurden, Jugoslawen, Roma und Sinti... angesprochen fühlen und HipHop praktizieren (...). [D]ie Message sollte klar sein: (...) Wir gehen unseren eigenen Pfad! ‘16

\section{Postethnische Perspektiven: HipHop als politische Praxis?}

Kanak Attak ist aus dieser Tradition entstanden. Dass die Gruppe in den Medien und der mehrheitsdeutschen linken Szene gerne als intellektuelle Kopfgeburt dargestellt wird, hat sicher viel mit der deutschen Auseinandersetzung mit Postcolonial Studies, Queer Theory und Subaltern Studies zu tun. So sie denn überhaupt stattfindet, ist sie meist streng akademisch, ohne Bezug zur bundesdeutschen Realität. ${ }^{17}$ Dass diese Theorien ursprünglich dazu dienten, praktische ,nicht-intellektuelle“ Widerstandsformen zu beschreiben, bleibt so weitestgehend unberücksichtigt, da die „Bezugs-Community“, um deren Widerstandspraxen es geht, nicht vorhanden ist (bzw. nicht als solche wahrgenommen wird). Das Modell Kanak Attak, dem die Kenntnis der genannten Theorien deutlich anzumerken ist, setzt sie dagegen in einen kritischen Bezug zu politischen Aktionsformen, so einerseits die Theorie-Praxis-Dichotomie zumindest ansatzweise überwindend, andererseits zu Formen greifend, die zu den Ursprüngen zurückführen, von denen sich die Theorie teilweise deutlich entfernt hat. Das Verhältnis zu diesen nicht traditionell linken Aktionsformen bleibt dabei allerdings ambivalent:

16 Die Zulu Nation wurde von Afrika Bambaataa, einem der „Väter“ des HipHop, 1973 als Selbsthilfe-Organisation für Jugendliche in der South Bronx gegründet. Inzwischen ist sie international aktiv und versucht, unter HipHop-Fans ein Bewusstsein der Geschichte und politischen Bedeutung (ihrer Version) der HipHop-Kultur zu verbreiten. Siehe: www.zulu-family.de.

17 Natürlich gibt es Ausnahmen von dieser Regel, so Breger 2000; siehe auch Quaestio 2000 und Gelbin et al. 1999. 
„Die Instrumente klassischer Antirassismuspolitik in Deutschland sind aus unserer Sicht gescheitert. Sie sind langweilig, bringen Leute nicht zusammen und wirken nicht gerade politisch motivierend (...). Wenn wir eine Maxi CD machen, machen wir das nicht, weil wir denken, es ist Pop und es ist hübsch, sondern wir wollen darüber Leute erreichen, die es nicht gewohnt sind oder es nicht besonders aufregend finden, in der taz unser Manifest zu lesen. Das heißt, es ist auch der politischen Situation geschuldet, dass man sich unterschiedlicher Instrumente bedient.“ (Ayata 1999: 3)

Die Ambivalenz ist verständlich: Einerseits bedingte die im Westen vorherrschende Priorität des schriftlichen Diskurses seine zentrale Stellung in der Konstruktion von Machtpositionen, den Ausschluss von Minderheiten aus seinem Kanon und die gleichzeitige Verlagerung von Widerstand auf nichtschriftliche Diskursformen. ${ }^{18}$ Andererseits erlauben kulturelle Aktionsformen - Musik, Tanz, Grafitti - die Identifikation von Minderheiten mit Körper, Emotion und Exotik. Die auf den ersten Blick vorhandenen Unterschiede hier intellektuelle, postmoderne, Kategorien hinterfragende Kanak AttakAktivistInnen, dort a-politische, anti-intellektuelle Macho-HipHopper - sind dennoch weniger absolut, als es scheinen mag. Wobei ich betonen muss, dass es mir hier nicht um HipHop als Kultur oder Rap als Musikrichtung geht, sondern um bestimmte Identitätsmodelle, die im deutschen HipHop entwickelt werden und die sich vom amerikanischen Vorbild lösen, indem sie sich auf die politische und soziale Situation in der Bundesrepublik beziehen und ebenso wie Kanak Attak eine offene, inklusive Gruppenidentität entwerfen, die eher auf einer gemeinsamen Interessenlage beruht als auf ethnischer Herkunft. Die Konstruktion von Identität über die Wiederaneignung einer unterdrückten, verdrängten Geschichte ist hier wie dort von zentraler Bedeutung, wird als Voraussetzung gesehen, der Fremdbestimmung und -kategorisierung durch die Mehrheitsgesellschaft offensiv begegnen zu können. Gleichzeitig geht es darum, diese Identität erstmals zu formulieren und über den Dialog zu problematisieren. ${ }^{19}$ Dazu Linguist von Advanced Chemistry:

„Es gibt in Deutschland Angehörige von Minderheiten. Es gibt Minderheiten mit deutschem Paß. Das bin ich zum Beispiel als schwarzer Deutscher. Es gibt Minderheiten in diesem Land ohne deutschen Paß. Das sind zum Beispiel Deutschtürken ich sage bewußt Deutschtürken - das sind Deutschjugoslawen, Deutschmarokkaner, wer auch immer. Aber wir gehören alle in einen Topf. Wir sind alle von Rassismus betroffen, nicht von Ausländerfeindlichkeit. Ich bin von Rassismus betroffen, habe aber das Glück, daß ich wenigstens nicht aus diesem Land rausgeschmissen werde.

18 Das ist natürlich eine sehr verkürzte Darstellung, die nicht behaupten will, dass es jemals eine homogene Position des Widerstands gab. Zu den Emanzipationsstrategien von Minderheiten im Westen gehörte immer auch der Versuch des Beweises, für eine Teilnahme am kanonisierten Diskurs qualifiziert zu sein.

19 Natürlich gibt es auch im deutschen HipHop andere Modelle, die explizit auf eine „türkische“, ,,italienische“ etc. Identität der der „Zweiten Generation“ angehörenden KünstlerInnen rekurrieren, genauso wie es politische MigrantInnenorganisationen gibt, die ethnische Identitätspolitik als Basis ihrer Arbeit sehen. 
Andererseits gibt es für mich keine Gesetze, die meine Rechte verteidigen als Minderheit" (Advanced Chemistry 1993: 16).

Dass die Eigendefinition der Objekte des Multikulturalismus-Diskurses bei ihren mehrheitsdeutschen Fürsprechern durchaus nicht unbedingt auf Begeisterung stößt, bekamen auch Advanced Chemistry zu spüren, nachdem sie „Fremd im eigenen Land“, das erste deutsche HipHop-Stück, das den hiesigen Alltagsrassismus gegen MigrantInnen und deutsche ethnische Minderheiten beschreibt, veröffentlicht hatten:

„Das Verrückte ist, daß wir natürlich einerseits von Leuten, die ideologisch entgegengesetzt, also rechts sind oder konservativ, angegriffen werden. Daß das denen natürlich nicht ins Bild paßt, wenn ein Schwarzer seinen grünen $\mathrm{Pa} ß$ in die Kamera hält und dann offensiv sagt, daß er Deutscher ist. Andererseits aber, und das ist das Verrückte daran: Leute, die sich für sehr links halten, glauben, daß wir uns dadurch distanzieren wollen von solchen Minderheiten in Deutschland, die keinen grünen $\mathrm{Pa}$ haben. Der Vorwurf kam sehr oft"“ (ebd.).

Ähnlich ablehnende bis herablassende Reaktionen auf explizit politische Rapper, inklusive dem „karitativen HipHop-Kollektiv“ Brothers Keepers (Musik Express), sind nach wie vor an der Tagesordnung. ${ }^{20}$ Sicher ist das zum Teil ein für die mehrheitsdeutsche Linke typisches Abgrenzen von Territorien, nach dem Motto: „Ihr könnt die Soul Rebels sein, aber die politische Analyse überlasst bitte uns." Letztendlich geht es aber auch hier um den Versuch, am tradierten Diskurs einer homogenen deutschen Identität festzuhalten, der die ebenso homogene Gruppe der „Ausländer“ gegenübergestellt wird. Da das vorherrschende Nationalitätsmodell auf klare Grenzen angewiesen ist, wird der beträchtlichen Gruppe innerhalb der deutschen Bevölkerung, die auf der Basis von legalen und/oder sozialen Konstruktionen von „Deutschsein“ ausgeschlossen bleibt, eine „Fremdheit“ zugeschrieben, die die durch diese künstliche Trennung hervorgerufenen Spannungen aus der Mitte der Gesell-

20 Brothers Keepers, auf Initiative von Adé und Don Abi Odukoya gegründet, ist ein Zusammenschluss einiger der populärsten afro-deutschen Rapper. Mit dem Song „Adriano (Letzte Warnung)“ - dem von Skins ermordeten Alberto Adriano gewidmet - der Widerstand gegen rassistische Gewalt ankündigt, erreichten sie nicht nur die Spitze der Charts, sondern schafften es auch, die strikte Trennung zwischen kulturellem Hybrid-Hype auf der einen und politischer Ausgrenzung von Nicht-Mehrheitsdeutschen auf der anderen Seite zu durchbrechen. „Letzte Warnung“ thematisiert explizit Rassismus in Deutschland aus der Sicht der Betroffenen. Das ist zwar nichts Neues, Fresh Familee, Skills en Mass oder Afrob haben das schon früher getan. Neu ist aber, dass es den im Projekt zusammengeschlossenen Künstlern explizit darum ging, ihre Popularität zu nutzen, um in die politische Debatte einzugreifen (und zwar über die musikalische Ebene hinaus, so ist u.a. kontinuierliche Jugendarbeit geplant) - und dass der Plan aufging: Das Medienecho übertraf das übliche Interesse an politischem HipHop bei weitem. Das hat mit der Popularität der Beteiligten zu tun und dem zunehmenden mainstream-Erfolg von HipHop. Es spiegelt vielleicht aber auch ein sich wandelndes deutsches Nationalbewusstsein wider. 
schaft auf die Körper derjenigen projiziert, die das Bild stören. Die Brüche bleiben bestehen, sollen aber gefälligst von den Betroffenen selbst gekittet werden. So werden in der juristischen Definition von Nationalität in Deutschland geborene „Ausländer“ und deutsche ethnische Minderheiten voneinander getrennt, denn auf dieser Ebene ist es von entscheidender Bedeutung, ob man die Staatsangehörigkeit seines Geburts- und Heimatlandes besitzt oder nicht. Hinzu kommt jedoch die soziale Definition von Zugehörigkeit, die bestimmt, wer im Alltag als deutsch wahrgenommen wird und wie das öffentliche Bild von „Deutschland“ und nationale Identität konzeptualisiert werden. Diese soziale Ebene kann, aber muss nicht mit juristischen Normen übereinstimmen. Generell ist es nach wie vor ein auf gewissen physischen Merkmalen beruhendes Bild vom Deutschen, das bestimmt, wer als solcher wahrgenommen wird, unabhängig von der tatsächlichen Nationalität. Umgekehrt bewirkt der Besitz eines deutschen Passes keineswegs die soziale Akzeptanz derjenigen, denen diese physischen Merkmale fehlen. Es gibt wohl keine afro-deutsche HipHop Crew, die diese Erfahrung nicht thematisiert hätte. Samy Deluxe, Rapper aus dem bürgerlichen Stadtteil Eppendorf in Hamburg:

„Ihr wisst, ich hörte schon im Kindergarten Weisse zu mir Nigger sagen.

Jetzt nach all den Jahren wird es Zeit den Scheiss zu hinterfragen.

Denn, wenn ich mich aufregte, war für sie die Sache klar:

,Du bist doch kein echter Nigger, Sam, du hast doch glattes Haar!'

Aha, die Farbe reichte aus, um mich zu beschimpfen.

Doch sie wunderten [sic], dass es mich traf und es traf mich am schlimmsten.

Viele hier verstehen dies Gefühl nicht im geringsten,

doch ich will, dass es alle kapier'n, sogar die dümmsten.

Wie oft warst denn du schon der einzig Weisse im Raum voller Schwarzer?

Noch niemals, das bezweifle ich kaum.

Und wie oft bist du kreuz und quer durchs Land gefahrn

und egal wo du hinkamst, fingen sie an dich anzustarrn. “

(Samy Deluxe feat. Brooke Russell, DJ Desue, „Sag mir wie es wär“ auf: Brothers

Keepers, Lightkultur, Album 2001)

Das gleichzeitige Vorhandensein von Kennzeichen des „Deutschen“ und des „Fremden“ ist inakzeptabel und muss daher diskursiv negiert werden. Diejenigen, die diese Simultanität auszeichnet, werden für das Lösen des untragbaren Widerspruchs verantwortlich gemacht; indem sie ihre „Fremdheit" assimilieren, sie als unabänderlich akzeptieren, den hippen Hybriden spielen, indem sie, kurz gesagt, eingestehen, dass sie ein ungekanntes, unerklärtes und unmögliches Phänomen darstellen.

Diese Kategorisierung erlaubt das Fortbestehen illusorischer Konzepte von unambivalenten, reinen und statischen nationalen Identitäten - im Extremfall durch die physische Entfernung derjenigen, die zwischen die angeblich klaren Kategorien fallen. ${ }^{21}$ Dennoch wird das diskursive Niemandsland

21 Die Abschiebung in ein „Herkunftsland“, das sie noch nie gesehen haben, kann in Deutschland geborene „Ausländer" theoretisch jederzeit treffen und durchaus 
zwischen den Grenzen seit Jahrzehnten, wenn nicht Jahrhunderten, von Menschen bewohnt, die sich zwischen sich angeblich ausschließenden Identitätskonzepten bewegen und eine Tradition schaffen, die zwar marginal, aber keineswegs unsichtbar ist. Ein notwendiger erster Schritt zur Etablierung eines einschließenden Modells nationaler Identität - und zwar legal und sozial - ist daher die Wiederentdeckung dieser Geschichte ethnischer Diversität, die die Künstlichkeit nicht hybrider, sondern „reiner“ Kulturen offenbart. Spätestens seitdem dem Projekt „Brothers Keepers“ enormes, wenn auch nicht immer wohlwollendes Medieninteresse entgegenschlägt, zeigt sich, dass HipHop nicht nur das Potenzial hat, diejenigen zu mobilisieren, über die zwar viel geredet wird, denen man aber nicht zuhört, sondern auch, genau dieses Zuhören von Seiten der Mehrheit zu erzwingen.

\section{Ausblick: Chancen und Gefahren}

Die Ausdrucksmöglichkeiten, die HipHop für bisher weitgehend mundtot gemachten Minderheiten geschaffen hat, sind in ihrer Wirkung nicht zu unterschätzen. Nicht nur, weil diese Minderheiten nicht mehr problemlos aus dem öffentlichen Diskurs ausgeschlossen und in die Rolle des stummen Objekts (je nach Interessenlage als Sündenbock oder Opfer präsentiert) gedrängt werden können, sondern gerade auch, weil HipHop einen Dialog von Minderheiten ermöglichen kann, ohne dass der regulierende und kontrollierende Umweg über Mehrheitsdeutsche in Kauf genommen werden muss. Und in diesem Dialog liegt die erfolgversprechendste Möglichkeit, sich gegen den europaweiten Aufschwung rechtsextremer, rassistischer, antisemitischer, antiislamischer und xenophober Kräfte zur Wehr zu setzen (und nur eine derartige Koalition bietet die Möglichkeit, aus einer Position der Stärke heraus Bündnisse mit anderen, auch mehrheitsdeutschen Gruppen einzugehen).

Der Erfolg birgt aber auch Gefahren. Die postmoderne EntertainmentKultur bedient sich exzessiv bei Subkulturen, die inzwischen zum Inbegriff von coolness geworden sind. Mit dieser Pseudo-Offenheit bestätigt sich der mainstream wieder als Maß aller Dinge, während VertreterInnen der Subkulturen selbst weiter über einen Mangel definiert werden: Subkultureller Chic kann eben nur von der „Hochkultur“ getragen werden. Subversives Potenzial wird so zum Verkaufsmittel domestiziert. In Deutschland sind VertreterInnen von Minderheiten erstmals massiv in der mainstream-Kultur präsent und haben die Chance, Inhalte selbst zu bestimmen. Die Präsenz beschränkt sich jedoch noch weitestgehend auf den Entertainment-Bereich, in dem Minderheiten traditionell zu einer Gratwanderung zwischen Reduzierung auf systemer-

nicht nur, wenn sie straffällig geworden sind. Jährlich werden Dutzende, wenn nicht Hunderte in Deutschland geborene Kinder und Jugendliche in ihnen völlig unbekannte Länder abgeschoben, weil ihre Eltern den Aufenthaltsstatus verlieren. 
haltende Stereotypen und einziger Repräsentationsmöglichkeit gezwungen sind. Tyron Ricketts, Schauspieler, Rapper und Produzent, beschreibt in „Afro-deutsch“ den Versuch, das subversive Potenzial dieser Situation auszuschöpfen:

„Scheiß auf die Galeere - ab jetzt mach ich Karriere!

mach den Carl Lewis Läufer, den Eddy Murphy Imitierer, mime den Drogendealer, spiele den Basketball- und Footballspieler (...)

Der Positivrassismusmeister nutzt Klischees zu seinem Vorteil, macht sie zu seinem Style.“

(Tyron Ricketts, „Afro-deutsch“, auf: Brothers Keepers 2001)

Gefährlich wird es dann, wenn die Klischees nicht mehr benutzt, sondern geglaubt werden und etwa afro-deutsche Mittelklasse-Rapper die ,dickhosige, posige Dickeier Macho-Nummer" (MC Nina) verinnerlichen, die (von) ihnen als authentische schwarze Gettokultur verkauft wurde. Das bedeutet nicht nur einen Verrat an der Tradition des afro-deutschen Widerstands, der in den letzten zwanzig Jahren zu einem großen Teil von denjenigen getragen wurde, die im HipHop gern unter „schwule Mädchen“ subsumiert werden. ${ }^{22}$ Es heißt auch, sich an der Instrumentalisierung von Minderheiten zu beteiligen, denen bestimmte reaktionäre Strukturen unterstellt werden, die dann als ,authentisch" (und nicht etwa Teil eines allgemeinen konservativen backlash) definiert werden und so wiederum ihren Ausschluss aus dem aufgeklärten, westlichen Diskurs rechtfertigen.

HipHopper haben sich eine exponierte Position erkämpft, die eine Chance für die gesamte community of color bietet. Es ist zu hoffen, dass sie diese Chance nicht vertun, indem sie konservative Positionen einnehmen, die letztlich mainstream-Interessen dienen und die subversive und komplexe Tradition verleugnen, aus der sie kommen. Denn trotz aller problematischen Strukturen - die im Übrigen typisch sind für männlich geprägte Jugendkulturen, vor allem exzessiver verbaler Sexismus und Homophobie, und die noch problematischer werden, wenn sie in politischen Aktivismus einfließen - bleibt HipHop die wichtigste Artikulationsform „ethnischer Außenseiter“ und bislang der einzige Bereich, in dem die Essentialisierung, Ethnisierung und Gettoisierung Nicht-Mehrheitsdeutscher im öffentlichen Diskurs erfolgreich durchbrochen

22 Über diese „Schulhof-Coolheit“ im HipHop, die „Mädchen“ und „schwul“ als Synonym für alles benutzt, was nicht den eigenen Vorstellungen von abgeklärter Männlichkeit entspricht, machen sich die Hamburger „Fettes Brot“ in ihrem gleichnamigen Song lustig. Zentrales Element des HipHop ist eine explizite Intertextualität, in der nicht nur die Musik anderer KünstlerInnen gesampelt, sondern sich immer wieder, positiv wie negativ, aufeinander bezogen wird. Meist sind die ausgetragenen Konflikte nicht unbedingt ideologischer Natur, gerade zum Thema Sexismus und Homophobie findet aber in letzter Zeit eine vermehrte Auseinandersetzung statt. Siehe neben „Schwule Mädchen“ auch „Doppel X Chromosom"von Nina (Nikita, 2001). 
und eine dezidiert eigene Position eingebracht wird, die die gängigen Definitionen von „Deutschen“ und „Ausländern“ nachhaltig durcheinander bringt.

\section{Zitierte Literatur}

Advanced Chemistry (1993): „Schwarz“ verstehen wir politisch. Die afrodeutsche HipHop-Gruppe Advanced Chemistry über ihre Erfahrungen mit alläglichem Rassismus, in: die tageszeitung, 25. 03. 1993, 16

Althaus, Dieter/Kraus, Josef/Gauger, Jörg-Dieter/Grewe, Hartmut (2002): PISA und die Folgen. Neue Bildungsdebatte und erste Reformschritte, Sankt Augustin: Zukunftsforum Politik der Konrad-Adenauer-Stiftung Ayata, Imran (1999): „Heute die Gesichter, morgen die Ärsche“, Spex 11/99 Barth, Ariane (1991): „Hier steigt eine Giftsuppe auf“, Der Spiegel 42/91 , 14. 10. 1991

Bax, Daniel (2001): Abschwellender Straßenslang, die tageszeitung, 10.10.2001, S. X-XI

Bhabha, Homi K. (2000): Die Verortung der Kultur, Tübingen: Stauffenberg. Bojadzijev, Manuela (2001): Der Kanak Attak-Aha-Effekt und die Überwindung der antirassistischen Arbeitsteilung, in: aka 451, 07.06.2001, S. 14

Bojadzijev, Manuela/Vassilis Tsianos (1999): „Mit den besten Absichten. Spuren des migrantischen Widerstands", $i z 3 w 240$ (September 1999).

Breger, Claudia (2000): „,Gekreuzt ${ }^{\star}$ und queer. Überlegungen zur Rekonzeptualisierung von gender, Ethnizität und Sexualität“, in: Röttger, Kati/Heike Paul, Differenzen in der Geschlechterdifferenz, Berlin: Erich Schmidt Verlag.

Bronfen, Elisabeth (Hg.) (1997): Hybride Kulturen. Beiträge zur angloamerikanischen Multikulturalismusdebatte, Tübingen: Staufenberg.

Dierbach, Heike: Deutsche unter sich, in: die tageszeitung, 26.4.2002, S. 25.

El-Tayeb, Fatima (2001). „Germans, Foreigners, and German Foreigners. Constructions of National Identity in Early $20^{\text {th }}$ Century Germany“, in: Salah Hassan/Iftikhar Dadi (Hg.), Unpacking Europe. Towards a Critical Discourse, Rotterdam: NAI.

Fallaci, Oriana (2002): Die Wut und der Stolz, München: List.

„Fremd und Deutsch“, SpiegelReporter 2/2000, 25. 01. 2000, o.A.

„Gefährlich fremd“, Der Spiegel, 16/97, 14. 04. 1997, o.A.

Gelbin, Cathy S./Kader Konuk/Peggy Piesche (1999): AufBrüche. Kulturelle Produktionen von Migrantinnen, Schwarzen und jüdischen Frauen in Deutschland, Königstein: Ulrike Helmer Verlag.

Ha, Kien Nghi (1999): Ethnizität und Migration, Münster: Westfälisches Dampfboot.

Hall, Stuart/Dave Morley/Kuan-Hsing (1996): Critical Dialogues in Cultural Studies, London: Routledge. 
Herbert, Ulrich (2001): Geschichte der Ausländerpolitik in Deutschland. Saisonarbeiter, Zwangsarbeiter, Gastarbeiter, Flüchtlinge, München: Beck.

Kanak Attak (1998): Manifest Kanak Attak und Basta! Verfügbar im Internet: http://www.kanak-attak.de

Kelly, Robin D. G. (1997): Yo' Mama's Disfunctional! Fighting the Culture Wars in Urban America, Boston: Beacon Press.

Kresta, Edith, „Bastarde gewinnen!“, die tageszeitung, 20. 08. 2001

Menrath, Stefanie (2001). Represent What... Performativität von Identitäten im HipHop, Hamburg: Argument.

Motte, Jan/Rainer Ohliger/Anne von Oswald (Hg.) (1999). 50 Jahre Bundesrepublik. 50 Jahre Einwanderung. Nachkriegsgeschichte als Migrationsgeschichte, Frankfurt/Main: Campus.

Müller, Alexander, Die neue linke Lässigkeit, die tageszeitung , 29. 05.1999

Quaestio (Hg): Queering Demokratie. Sexuelle Politiken. Berlin: Querverlag.

Schröder, Ralf, „Warum früher alles besser war“, Jungle World, 48/2000, 22. 11. 2000.

„Unterschriftenaktion zur Ausländerpolitik Union macht gegen Bonn mobil. Stoiber: Doppelte Staatsbürgerschaft gefährlicher als RAF“, Süddeutsche Zeitung, 04. 01. 1999, 5.

Auswahl-Discographie

Absolute Beginner

Flashnizm, Album 1996

Bambule, Album 1998

Bambule Remix / Boombule, Album 2000

Searching For The Jan Soul Rebels, Album 2001 (Eißfeldt solo)

Minidisco, Album 2001 (Denyo 77 solo)

Advanced Chemistry

Fremd im eigenen Land, Single 1992

Advanced Chemistry, Album 1995

Afrob

Rolle mit HipHop, Album 1999

Made in Germany, Album 2001

Brothers Keepers

Adriano (Letzte Warnung), Single 2001

Lightkultur, Album 2001

Cora E.

Und der MC ist weiblich, Album 1998 
Fettes Brot

Auf einem Auge blöd, Album 1995

Außen Top Hits, innen Geschmack, Album 1996

Fettes Brot läßt grüßen, Album 1998

Für die Welt, Album 2000

Schwule Mädchen, Single 2001

Fresh Familee

Falsche Politik, Album 1993

Alles frisch, Album 1994

Wir sind da, Album 1997

Freundeskreis

Quadratur Des Kreises, Album 1997

Esperanto, Album 1999

En Directo, Album 2000

Nina

Nikita, Album 2001

Samy de Luxe

Deluxe Soundsystem, Album 2000

Samy deluxe, Album 2001

Skills en Masse

Meli, Album 2001 